\title{
Symmetry considerations in the quasi-static approximation of volume conductor theory
}

\author{
$\mathrm{J}$ C de Munck $\dagger$ and $\mathrm{B}$ W van Dijk $\ddagger$ \\ $\dagger$ Twente University of Technology, PO Box 217, 7500 AE Enschedee, The Netherlands \\ $\ddagger$ The Netherlands Ophthalmic Research Institute, PO Box 12141, 1100 AC Amsterdam, \\ The Netherlands
}

Received 9 July 1980, in final form 19 December 1990

\section{Introduction}

In living subjects electromagnetic signals are generated which can be measured electrically with electrodes and normal amplifiers or magnetically, by means of SQUIDmagnetometers. The former technique is called EEG (electro-encephalography), the latter MEG (magneto-encephalography). Since the electromagnetic field patterns are dependent on physiological processes inside the body, a study of the electromagnetic field can help to understand these physiological processes. In this note some theoretical problems which are posed by such a study are considered. The theoretical problems imply questions such as 'How does the measured electromagnetic field depend on the underlying generators and on the medium in which these generators are embedded?', 'Are there sources which produce no magnetic field and under which conditions does this happen?', 'Are there advantages of magnetic rather than electric measurements?'.

Before answering these questions the way in which the electromagnetic field is generated should be considered. At a microscopic level, there are different physical mechanisms responsible for the generation of the electromagnetic field. Inside the brain, there are the synaptic interactions of neurons which produce the EEG and MEG. Cardiac potentials and cardiac magnetic induction are generated by the synchronous polarization of cardiac muscle cells. A third example is the electromagnetic field observed at the limbs. This field is caused by compound action potentials, which travel through the peripheral nerves by axonal transport. The main assumption of volume conductor theory is that, at a macroscopic level, all these electromagnetic effects can be described by current sources, embedded in a conducting (and possibly polarizable) medium. With this assumption the relationship between the current source density function and the resulting potential and magnetic induction can be made explicit. Another consequence is that the physical mechanisms underlying E'EG and MEG are identical.

In section 2 of this note we will briefly review the mathematical physical theory of biological volume conduction. The relationship between current sources, medium properties and the electric and magnetic induction is derived from Maxwell's equations, together with some material equations. How the resulting equations can be solved will not be considered. Instead, some useful properties of the solutions will be derived in section 3 . 


\section{Derivation of the volume conductor equations from Maxwell's equations}

Although the equations governing the electromagnetic fields in living tissue have been derived previously, (e.g. Barnard et al 1967, Geselowitz 1967), it is meaningful to look at them again. It appears, for instance, to be possible to derive from Maxwell's equations an equation for the magnetic induction, which is independent of the electric field, without assuming that the quasi-stationary approximation is valid. Moreover, it is not necessary to assume that the polarization of the medium vanishes to make the equations for the electromagnetic field solvable.

\subsection{Maxwell's equations}

The macroscopic Maxwell equations are an (incomplete) system of partial differential equations, which describes the electromagnetic fields in a conducting, polarizable and magnetizable medium. These equations are given by, for example, Jackson (1962):

$$
\begin{aligned}
& \operatorname{div} \boldsymbol{D}=\rho \\
& \operatorname{curl} \boldsymbol{H}-\partial \boldsymbol{D} / \partial t=\boldsymbol{J} \\
& \operatorname{div} \boldsymbol{B}=0 \\
& \operatorname{curl} \boldsymbol{E}+\partial \boldsymbol{B} / \partial t=0 .
\end{aligned}
$$

Here, $\boldsymbol{D}$ is the dielectric displacement, $\rho$ is the charge density, $\boldsymbol{H}$ is the magnetic field, $\boldsymbol{J}$ is the total current density, $\boldsymbol{B}$ is the magnetic induction and $\boldsymbol{E}$ is the electric field. Maxwell's equations are incomplete without the material equations, which specify the relationship between, on the one hand, the dielectric displacement and the electric field, and, on the other hand, the magnetic field and the magnetic induction. For biological tissues it is assumed that $\boldsymbol{D}$ and $\boldsymbol{H}$ depend linearly on the local electric field and magnetic induction respectively. This dependence may, however, be anisotropic, so that the directions of the corresponding fields are not necessarily equal:

$$
\begin{aligned}
& \boldsymbol{D}=\boldsymbol{\epsilon} \boldsymbol{E}=\varepsilon_{0}\left(\mathbf{I}+\boldsymbol{\chi}_{1}\right) \boldsymbol{E} \\
& \boldsymbol{H}=\boldsymbol{\mu}^{\text {inv }} \boldsymbol{B}=\left(1 / \mu_{0}\right)\left(\mathbf{I}+\boldsymbol{\chi}_{\mathrm{m}}\right)^{\text {inv } B}
\end{aligned}
$$

Here, $I$ is the identity tensor, and $\varepsilon_{0}$ and $\mu_{0}$ are the permittivity and magnetic permeability of free space respectively. Equation (5) expresses how strongly the neutral particles, which constitute the medium, polarize when an electric field is applied. Equation (6) gives the effect of the magnetic field on the magnetic dipole moment of the medium. This effect is neglected, generally, so that $\chi_{\mathrm{m}}=0$. This assumption is, however, not essential for deriving the basic equations of a volume conductor, as will be shown.

In order to obtain a complete system of equations, one extra equation is necessary which describes the relationship between $\boldsymbol{J}, \boldsymbol{E}$ and the impressed current, $\boldsymbol{J}_{\mathrm{i}}$. The latter represents the actual generators of the electromagnetic fields. The total current density, $\boldsymbol{J}$, equals the sum of the impressed current and the volume current, which is caused by the electromagnetic forces acting on the free charges (electrons as well as ions) in the medium. This volume current is again assumed to be linearly dependent on the local electric field. In a formula, we have

$$
\boldsymbol{J}=\boldsymbol{\sigma} \boldsymbol{E}+\boldsymbol{J}_{\mathrm{i}} \text {. }
$$

In equation (7) the conductivity $\boldsymbol{\sigma}$ tensor is used to express anisotropic conduction. This type of conduction has been demonstrated for some tissues (Rush and Driscoll 1968, Nicholson and Freeman 1975). 
Now, we face the problem of expressing the electromagnetic fields in terms of the generator, $\boldsymbol{J}_{\mathrm{i}}$. With equations (1) to (7) a partial differential equation can be derived for the magnetic induction, in which the other fields do not appear. To this end we note that all equations are linear and time invariant, so that it makes sense to consider only time functions of the type $e^{j \omega t}$. The symbols $\boldsymbol{B}, \boldsymbol{E}, \boldsymbol{H}$, etc, then represent the local complex amplitude of the corresponding fields, at the circle frequency, $\omega$. Other functions can be obtained from the superposition of sources with different frequencies.

With equations (2) and (5)-(7) we find for the magnetic field

$$
\operatorname{curl}\left[\boldsymbol{\gamma}^{\mathrm{inv}}\left(\operatorname{curl} \boldsymbol{\mu}^{\mathrm{inv}} \boldsymbol{B}\right)\right]+j \omega \boldsymbol{B}=\operatorname{curl}\left(\boldsymbol{\gamma}^{\mathrm{inv}} \boldsymbol{I}\right)
$$

For the electric field there are two equations:

$$
\begin{aligned}
& \operatorname{div}(\boldsymbol{\gamma} \boldsymbol{E})=-\operatorname{div} \boldsymbol{J}_{\mathrm{i}} \\
& \operatorname{curl} \boldsymbol{E}=-j \omega \boldsymbol{B}
\end{aligned}
$$

where $\boldsymbol{\gamma}$ is defined as

$$
\gamma(\omega)=\boldsymbol{\sigma}+j \omega \varepsilon
$$

In principle, the magnetic induction can be calculated from equation (8) and the result can be used to find the electric field, equations (9) and (10). These equations neither depend on the conductivity nor on the polarizability separately, but only on the combination (equation (11)). Therefore, both these material properties can be expressed by one function, $\gamma$, which depends on the circle frequency, $\omega$, and on the position, $x$. If this function is expanded in a Taylor series with respect to $\omega$, the constant term represents the $(D C)$ conductivity and the linear coefficient corresponds to the polarizability. Although the physical interpretation of higher-order terms in $\omega$ is less obvious, there are no theoretical objections against including them. Furthermore, it is clear that previous results, where the polarizability of the medium has not been taken into account, remain valid if all fields are considered as a complex amplitude dependent on $\omega$. In the following, $\gamma$ will be referred to as the conductivity.

\subsection{The quasi-static approximation}

If all terms of the order $j \omega \boldsymbol{B}$ can be neglected, the system of differential equations becomes much simpler, since, in that case curl $\boldsymbol{E}=0$, and hence

$$
\boldsymbol{E}=-\operatorname{grad} \psi
$$

where $\psi$ is the electric potential. Equation (9) becomes the impedance equation

$$
\operatorname{div}(\boldsymbol{\gamma} \operatorname{grad} \psi)=\operatorname{div} \boldsymbol{J}_{\mathrm{i}}
$$

and equation ( 8 ) becomes

$$
\operatorname{curl}\left[\gamma^{\text {inv }}\left(\operatorname{curl} \mu^{\text {inv }} B\right)\right]=\operatorname{curl}\left(\gamma^{\text {inv }} J_{i}\right) .
$$

In this way the electric and the magnetic field have been completely separated and the resulting equations are much easier to solve. Another consequence is that in this (quasi-static) approximation the electric field, and hence the potential, are independent of the permittivity tensor, $\mu$. Without the approximation, this dependence exists through equations (8) and (10). 
We will now consider under which circumstances the time-varying magnetic induction may be neglected. Assume that $\gamma$ and $\mu$ are constant scalars. Then equation (8) becomes (using equation (3) and curl curl $\boldsymbol{B}=\operatorname{grad} \operatorname{div} \boldsymbol{B}-\Delta \boldsymbol{B}$ )

$$
\Delta \boldsymbol{B}=j \omega \gamma \mu \boldsymbol{B}-\mu \operatorname{curl} \boldsymbol{J}_{\mathrm{i}} .
$$

The solution of this equation is

$$
\boldsymbol{B}(\boldsymbol{x})=-\frac{\mu}{4 \pi} \iiint_{-\infty}^{+\infty} \frac{\operatorname{curl}\left(J_{i}\left(\boldsymbol{x}^{\prime}\right)\right) \mathrm{e}^{\mathrm{i} k(\omega)|x-x|}}{\left|\boldsymbol{x}-\boldsymbol{x}^{\prime}\right|} \mathrm{d} \boldsymbol{x}^{\prime}
$$

in which the square of the amplitude of the wave vector, $k$, equals $k^{2}=|\omega \gamma \mu|=$ $\omega \mu\left(\sigma^{2}+\omega^{2} \varepsilon^{2}\right)^{1 / 2}$. The physiologically interesting range of frequencies is below $100 \mathrm{~Hz}$, and so $\omega<620 \mathrm{rad} \mathrm{s}^{-1}$. Furthermore, it has been found that for most living tissue $\varepsilon<10^{6} \varepsilon_{0}$ (Pethig and Kell 1987), $\sigma<0.7 \Omega^{-1} \mathrm{~m}^{-1}$ (Geddes and Baker 1967) and $\mu \simeq \mu_{0}$. With these values it can be calculated that $(\sigma \mu \omega)^{1 / 2}<5 \times 10^{-2} \mathrm{~m}^{-1}$ and $(\varepsilon \mu \omega)^{1 / 2}<$ $10^{3} \omega / c<2 \times 10^{-3} \mathrm{~m}^{-1}$. Since the distances involved are much smaller than $20 \mathrm{~m}$, we have $k\left|x-x^{\prime}\right| \ll 1$. The exponential function in equation (16) therefore approaches unity, and hence this solution tends to the solution of the simplified equation (14). The same conclusion has been reached by Plonsey and Heppner (1967) following a slightly different reasoning. They analyzed propagation, capacitance and induction effects separately and therefore their derivation is more complicated.

\section{Symmetry properties in the quasi-static approximation}

A vanishing magnetic induction oxtside a conductor may be considered as the result of symmetry, as shown in this section. The quasi-static approximation will be assumed to be valid and the magnetic permeability to be constant and isotropic. If $\boldsymbol{R}$ is an orthogonal matrix, then symmetries of scalars, vectors and tensors can be defined as follows.

A scalar, $s(\boldsymbol{x})$, is called symmetric under $\mathbf{R}$ if

$$
s(\boldsymbol{x})=s(\mathbf{R} \boldsymbol{x}) \quad \text { for all } \boldsymbol{x} \in \mathbb{R}^{3} .
$$

A vector field, $\boldsymbol{B}(\boldsymbol{x})$ is called symmetric under $\mathbf{R}$ if

$$
\boldsymbol{R B}(\boldsymbol{x})=\boldsymbol{B}(\mathbf{R} \boldsymbol{x}) \quad \text { for all } \boldsymbol{x} \in \mathbb{R}^{3}
$$

and a tensor field, $\gamma(x)$, is symmetric if

$$
\mathbf{R} \gamma(x) \mathbf{R}^{-1}=\gamma(\mathbf{R} \boldsymbol{x}) \quad \text { for all } x \in \mathbb{R}^{3} .
$$

These definitions are trivial for scalar functions and vector fields. The meaning of equation (19) can be understood easily if $\gamma$ represents a conductivity tensor (i.e. if $\boldsymbol{\gamma}(\boldsymbol{x})$ is a positive matrix for every $\boldsymbol{x}$. In that case $\boldsymbol{\gamma}$ has three orthogonal eigenvectors and equation (19) states that if $e$ is an eigenvector at the position $x$ (i.e. $\gamma(x) e=\lambda e$ ), then $\mathbf{R} \boldsymbol{e}$ will be an eigenvector at the point $\mathbf{R} \boldsymbol{x}$, with the same eigenvalue (so, $\boldsymbol{\gamma}(\mathbf{R} \boldsymbol{x}) \mathbf{R} \boldsymbol{e}=$ $\boldsymbol{R} \gamma(\boldsymbol{x}) \boldsymbol{e}=\lambda \boldsymbol{R} \boldsymbol{e})$. Since $\boldsymbol{\gamma}$ is uniquely defined by its eigenvectors and eigenvalues, equation (19) completely describes the symmetry.

The term cylinder symmetry will be used if a function is symmetric for every rotation about a given axis (commonly the $z$ axis). A spherically symmetric function is symmetric 
for every rotation about a given point (commonly the origin). Functions evaluated at a transformed point will be denoted by a prime (e.g. $\psi^{\prime}(\boldsymbol{x})=\psi(\mathbf{R} x)$ ).

Given these definitions it can be proven that the potential, $\psi(\boldsymbol{x})$, will be symmetric if the impressed current, $\boldsymbol{J}_{i}$, and the conductivity, $\gamma$, are symmetric. First, it will be assumed that the impedance equation (equation (13)) has only one unique solution (up to a constant). Then we only have to prove that if $\psi$ is a solution, so is $\psi^{\prime}$.

We find

$$
\begin{aligned}
\operatorname{div}\left(\boldsymbol{g r a d} \psi^{\prime}\right) & =\operatorname{div}\left(\boldsymbol{\gamma} \mathbf{R}^{-1} \operatorname{grad}^{\prime} \psi^{\prime}\right) \\
& =\operatorname{div}\left(\mathbf{R}^{-1} \boldsymbol{\gamma}^{\prime} \operatorname{grad}^{\prime} \psi^{\prime}\right) \\
& =\operatorname{div}^{\prime}\left(\boldsymbol{\gamma}^{\prime} \operatorname{grad} \psi^{\prime}\right)=\operatorname{div}^{\prime} \boldsymbol{J}_{\mathrm{i}}^{\prime} \\
& =\operatorname{div}^{\prime}\left(\mathbf{R R}^{-1} \boldsymbol{J}_{\mathrm{i}}\right)=\operatorname{div} \boldsymbol{J}_{\mathrm{i}}
\end{aligned}
$$

which shows that $\psi$ and $\psi^{\prime}$ are solutions of the same equations, and hence they must be identical. In the derivation the following have been used: $\operatorname{grad}^{\prime}(\cdot)=\mathbf{R}^{-1} \operatorname{grad}(\cdot)$, and $\operatorname{div}^{\prime}(\cdot)=\operatorname{div}(\mathbf{R} \cdot)$. The result has been obtained before (de Munck et al 1988), and corresponds to Bloch's theorem used in solid-states physics (Ashcroft and Mermin 1976).

To generalize the conditions for which the magnetic induction is independent of the conductivity function, it is not sufficient to claim that $\gamma$ and $\boldsymbol{J}_{\mathrm{i}}$ have cylinder symmetry. Sufficient conditions can be specified if it is noted that $\gamma$ defines (since it is a positive symmetric tensor) for every $x \in \mathbb{R}^{3}$ three orthonormal vectors (the principal directions), and three corresponding positive numbers (the principal conductivities). We will prove that if the volume conductor meets the following conditions:

(1) $\gamma$ has cylinder symmetry;

(2) one of the principle directions of $\gamma$ parallels the $\hat{\varphi}$-direction; and

(3) $\boldsymbol{J}_{i}$ has cylinder symmetry;

then $B$ outside $V$ is independent of the conductivity. Note that if the conductor is isotropic, it automatically meets condition (2). The $\hat{\varphi}$ direction is the direction of increasing $\varphi$, if cylinder coordinates are used (see figure 1 ).

If the impressed current satisfies conditions (3) and (4):

(4) the $\hat{\varphi}$ component of $J_{i}$ vanishes;

then the magnetic induction is zero outside a conductor satisfying conditions (1) and (2).

With the conditions given in $\S 2$ it follows that

$$
\text { curl } B=-\mu_{0} \gamma \operatorname{grad} \psi+\mu_{0} J_{\mathrm{i}}
$$

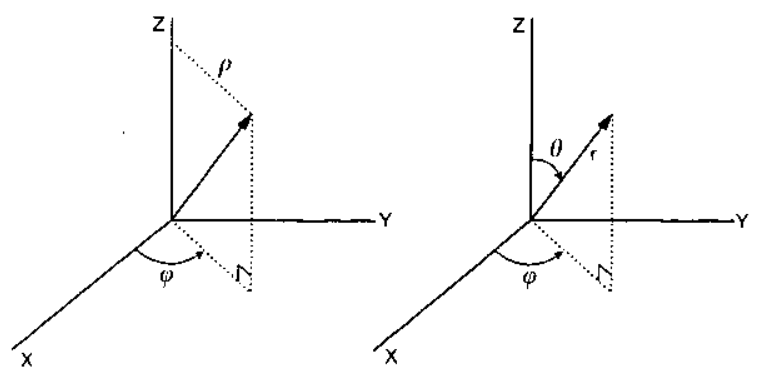

Figure 1. Coordinates. Left: cylindrical coordinates $(\varphi, \rho, z)$; right spherical coordinates $(r, \vartheta, \varphi)$. 
and $\operatorname{div} B=0$. The solution of equation (21) is then (Morse and Feshbach 1953)

$$
B(x)=\frac{\mu_{0}}{4 \pi} \iiint_{-\infty}^{+\infty} \frac{\operatorname{curl} J_{\mathrm{i}}-\operatorname{curl}(\gamma \operatorname{grad} \psi)}{\left|x-x^{\prime}\right|} \mathrm{d} x^{\prime}
$$

We can see that in a homogeneous and isotropic medium the second term in equation (22) vanishes, because $\operatorname{curl}(\operatorname{grad} \cdot)=0$. Therefore this term represents the contribution of the so-called 'secondary' sources, $\boldsymbol{B}_{\mathrm{sec}}$, which are induced by the medium inhomogeneities. The contribution of the 'primary' sources, $\boldsymbol{B}_{\infty}$, are represented by the first term of equation (22) and are independent of the conductivity.

Since $\varepsilon_{0} \mu_{0} \omega$ is very small, we conclude from equations (21) and (11) that

$$
\text { curl } B=0 \quad \text { for all } x \text { outside } V \text {. }
$$

Hence for $x$ outside the conductor a magnetic potential, $U(x)$, can be so defined that

$$
\boldsymbol{B}=\operatorname{grad} U
$$

Then, because $\boldsymbol{B}$ satisfies $\operatorname{div} \boldsymbol{B}=0$ and because of equation (23), $U$ is a solution of the following boundary value problem.

$(\operatorname{grad} U) \cdot n=\frac{\mu_{0}}{4 \pi} n(x) \cdot\left(\iint_{-\infty}^{+\infty} \int_{-\infty}^{\operatorname{curl} J_{i}} \frac{x^{\prime} \mid}{\mid x} x^{\prime}-\iiint_{-\infty}^{+\infty} \frac{\operatorname{curl}(\gamma \operatorname{grad} \psi)}{\left|x-x^{\prime}\right|} \mathrm{d} x^{\prime}\right)$

for all $x \in \partial V$

$U \rightarrow 0 \quad$ for $x \rightarrow \infty$

$\Delta U=0 \quad$ for $x$ outside $V$.

Here, $\partial V$ is the boundary of the conductor and $n$ is the outward normal to this surface.

We see that the magnetic induction outside the volume conductor depends on the curl of the primary and the secondary sources. At the end of this section it will be demonstrated that under conditions (1)-(3) the contribution of the secondary sources to the magnetic potential, $U$, vanishes, and under (3) and (4), the contribution of the primary sources vanishes. The main argument is that under these conditions, the magnetic induction only has a $\hat{\boldsymbol{\varphi}}$ component, and hence the inner product with $\boldsymbol{n}$ in equation (26) vanishes. Then, from conditions (1)-(3) it follows that

$$
\boldsymbol{B}_{\mathrm{sec}}(\boldsymbol{x}) \cdot \boldsymbol{n}(\boldsymbol{x})=0 \quad \text { for all } \boldsymbol{x} \in \partial \boldsymbol{V} \text {. }
$$

Since the solution, $U(\boldsymbol{x})$, of equation (25) is unique, it is independent of $\boldsymbol{\gamma}$. Therefore, the magnetic induction is also independent of $\boldsymbol{\gamma}$.

This result seems to imply great advantages in using magnetic induction recordings instead of electric potentials in inverse algorithms. The drawback is, however, that in a volume conductor satisfying conditiond (1) and (2), there are many sources which are undetectabie with magnetic measurements. This can be seen from equation (25). If a source satisfies conditions (3) and (4) then the contribution of the primary sources only has a $\hat{\boldsymbol{\varphi}}$ component. In that case the boundary condition on $\partial V$ becomes $\partial U / \partial \boldsymbol{n}=0$, and hence $U$ and $\boldsymbol{B}$ are identically zero, outside the conductor. An example of a situation for which conditions (1) and (4) are met is a radial dipole in a spherically symmetric volume conductor. 
To complete the proof we have to demonstrate that under conditions (1)-(3), and (3) and (4), the magnetic induction caused by respectively the secondary and the primary sources vanishes. For every vector field, $\boldsymbol{K}(\boldsymbol{x})$, the curl can be written in cylinder coordinates as

$\operatorname{curl} \boldsymbol{K}=\left(\frac{1}{\rho} \frac{\partial K_{z}}{\partial \varphi}-\frac{\partial K_{\varphi}}{\partial z}\right) \hat{\boldsymbol{\rho}}+\left(\frac{1}{\rho} \frac{\partial K_{\rho}}{\partial z}-\frac{\partial K_{z}}{\partial \rho}\right) \hat{\varphi}+\left(\frac{\partial}{\partial \rho}\left(\rho K_{\varphi}\right)-\frac{1}{\rho} \frac{\partial K_{\rho}}{\partial \varphi}\right) \hat{z}$

If $\boldsymbol{J}_{\mathrm{i}}$ is substituted for $\boldsymbol{K}$, it can be concluded from the assumed cylinder symmetry, condition (3), that the derivatives with respect to $\varphi$ vanish, and from condition (4) it follows that all components of curl $\boldsymbol{J}_{i}$, except the $\hat{\boldsymbol{\varphi}}$ component, are zero. Therefore, conditions (3) and (4) imply that $\boldsymbol{n} \cdot \boldsymbol{B}_{\infty}=0$, and hence the primary sources do not contribute to the magnetic induction outside the conductor.

Next, it will be shown that $\boldsymbol{\gamma} \cdot \operatorname{grad} \psi$ has cylinder symmetry and has a vanishing $\hat{\varphi}$ component. Then, by the same argument as was used for the primary sources, it can be shown that the magnetic induction caused by the secondary sources only has a $\hat{\varphi}$ component. From condition (3) and equation (20) it follows that the potential has cylinder symmetry. Hence we have

$$
\gamma \operatorname{grad} \psi=\mathbf{R}^{\text {inv }} \boldsymbol{\gamma}(\mathbf{R} \boldsymbol{x}) \mathbf{R R}^{\text {inv }} \operatorname{grad}^{\prime} \psi(\mathbf{R} \boldsymbol{x})=\mathbf{R}^{\text {inv }} \boldsymbol{\gamma}^{\prime} \operatorname{grad}^{\prime} \psi^{\prime}
$$

which demonstrates the required symmetry.

Finally, it will be shown that condition (2) results in a vanishing $\hat{\varphi}$ component for secondary sources. As was stated earlier, the fact that $\gamma(x)$ is positive means that the conductivity tensor has three orthogonal eigenvectors with positive eigenvalues, at every point in space. Condition (2) states that one of these vectors equals $\hat{\varphi}$. It follows that a 'multiplication' of $\boldsymbol{\gamma}$ by any vector which is a linear combination of $\hat{\boldsymbol{\rho}}$ and $\hat{\boldsymbol{z}}$ will result in another linear combination of these two unit vectors. Since $\psi$ has cylinder symmetry, $\operatorname{grad} \psi$ will be a linear combination of $\hat{\boldsymbol{\rho}}$ and $\hat{\vec{z}}$, and hence it follows that

$$
\hat{\varphi} \cdot(\gamma \operatorname{grad} \psi)=0
$$

and hence from equation (27) we have that $\boldsymbol{n} \cdot \operatorname{curl}(\boldsymbol{\gamma} \operatorname{grad} \psi)=0$. In this way the proof is completed, formally. In figure 2 a more intuitive representation of this result is given.

To summarize, two propositions have been proven: (i) under conditions (1), (2) and (3) the contribution of secondary sources to the magnetic induction outside a conductor vanishes; and (ii) under conditions (3) and (4) the primary sources do not contribute to this field. These propositions have implications for the existence of magnetically silent sources and the dependence on the conductivity of the magnetic induction outside the conductor.

The first proposition is based on condition (3), that the impressed current has cylinder symmetry. This assumption is not needed if the volume conductor is spherically symmetric. It has already been proven that in such a conductor the secondary sources are magnetically silent outside the conductor (Baule and McFee 1965, Grynszpan and Geselowitz 1973, Sarvas 1987), but these authors did also assume that the conduction was isotropic. These results will be generalized by including a specific class of anisotropy.

Note that a spherically symmetric volume conductor is completely specified by its radial and tangential conductivity functions, $\varepsilon(r)$ and $\eta(r)$. To show that the secondary scurces are silent, it has to be demonstrated that the radial component of $\operatorname{curl}(\gamma \operatorname{grad} \psi)$ vanishes. For this purpose the radial component of the curl and the gradient will be 

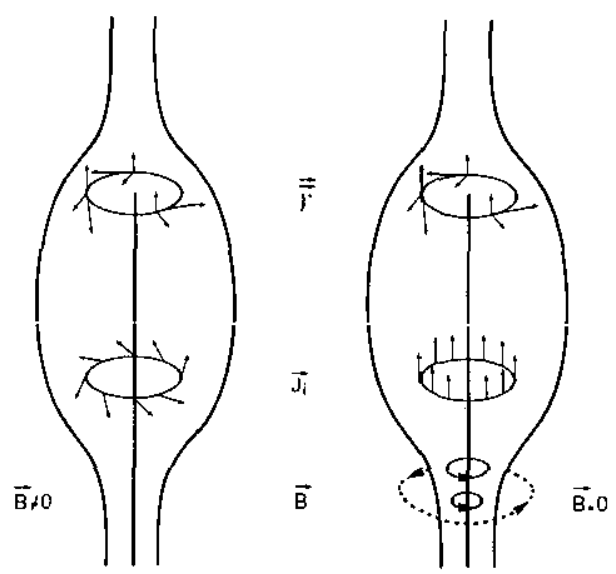

Figure 2. An intuitive explanation of silent primary and secondary sources. The 'stretched bottles' represent cylindrically symmetric volume conductors. The upper arrows in both figures give the principal directions of the conductivity tensor, $\gamma(\boldsymbol{x})$. For all $\boldsymbol{x}$, one of these directions parallels the $\hat{\boldsymbol{\varphi}}$ direction, and the other directions are arbitrary. If the conductor is isotropic, this condition is automatically satisfied. The middle set of arrows represent a circularly symmetric impressed current, $\boldsymbol{J}_{\mathrm{i}}$. In the left figure the $\hat{\boldsymbol{\varphi}}$ component of the current is arbitrary and therefore the resulting magnetic induction, $\boldsymbol{B}(\boldsymbol{x})$, is non-zero. However, the contribution of the secondary sources to this field is zero, and hence $B(x)$ is independent of $\gamma(x)$. In the right figure the $\hat{\varphi}$ component of the current vanishes and therefore $\boldsymbol{B}$ only has a $\hat{\varphi}$ component. Since curl $\boldsymbol{B}=0$ for $\boldsymbol{x}$ outside $V$, it follows that the magnetic induction vanishes for $\boldsymbol{x}$ outside $V$.

expressed in spherical coordinates:

$$
\hat{\boldsymbol{r}} \cdot \operatorname{curl} \boldsymbol{K}=\frac{1}{r \sin \vartheta}\left(\frac{\partial}{\partial \vartheta}\left(\sin \vartheta K_{\varphi}\right)-\frac{\partial K_{\vartheta}}{\partial \varphi}\right)
$$

and

$$
\operatorname{grad}()=\left(\frac{\partial}{\partial r}\right) \hat{\boldsymbol{r}}+\frac{1}{r}\left(\frac{\partial}{\partial \vartheta}\right) \hat{\boldsymbol{\vartheta}}+\frac{1}{r \sin \vartheta}\left(\frac{\partial}{\partial \varphi}\right) \hat{\boldsymbol{\varphi}} .
$$

If $\boldsymbol{\gamma} \cdot \operatorname{grad} \psi$ is substituted for $\boldsymbol{K}$ then we get, using equations (30) and (31)

$$
\begin{aligned}
\hat{\boldsymbol{r}} \cdot \operatorname{curl} \boldsymbol{K} & =\frac{1}{r \sin \vartheta}\left[\frac{\partial}{\partial \vartheta}\left(\frac{\sin \vartheta}{r \sin \vartheta} \eta(r) \frac{\partial \psi}{\partial \varphi}\right)-\frac{\partial}{\partial \varphi}\left(\frac{\eta(r)}{r} \frac{\partial \psi}{\partial \vartheta}\right)\right] \\
& =\frac{\eta(r)}{r^{2} \sin \vartheta}\left(-\frac{\partial^{2} \psi}{\partial \vartheta \partial \varphi}-\frac{\partial^{2} \psi}{\partial \varphi \partial \vartheta}\right)=0 .
\end{aligned}
$$

Therefore, the magnetic induction outside a spherically symmetric conductor is independent of the conductivity, no matter what current sources are present inside.

\section{Discussion}

It has become common knowledge that the magnetic field outside a spherical isotropic volume conductor generated by a radial current dipole is zero. For other sources it can be shown that the magnetic induction outside this kind of conductor is independent of the conductivity function. In this paper these results are generalized, by including anisotropy and cylinder symmetry (contrary to spherical symmetry).

The problem of finding the potential in a spherically symmetric volume conductor was solved analytically by de Munck (1988). This solutions was used by van Dijk et 
al (1990) to show that anisotropy of the skull and the cortex causes large systematic errors in dipole parameter estimates based on electric data. The results of the present paper imply that even in the case of anisotropic spherical shells, the magnetic induction is unaffected by the conductivity parameters. This seems to imply great advantages of using MEG instead of EEG. However, in this kind of volume conductor there are many sources which do not generate a magnetic field. A well-known example is a radial dipole, or a layer of radial dipoles. However, from the symmetry considerations of this paper, it follows that a circular array of tangential dipoles with a vanishing $\varphi$ component does not produce a magnetic field either.

The generalization to cylinder symmetry might be useful to study compound action potentials in limbs (Trahms et al 1989), or to calculate the magnetic field outside a single fibre (Plonsey 1981). It implies that a compound action potential, which travels precisely through the symmetry axis of a (cylindrical) limb, produces no magnetic field outside the limb. Another implication concerns the effect of inhomogeneities in the brain on the magnetic induction generated outside the head. If the inhomogeneities together with the head are approximately circular symmetric, and if the sources also obey this symmetry, then the magnetic field is unaffected by the inhomogeneities.

\section{References}

Ashcroft W and Mermin N D 1976 Solid State Physics (Philadelphia, PA: Holt, Rinehart and Winston) pp 132-9

Barnhard A C L, Duck I M and Lynn M S 1967 The application of electromagnetic theory to electrocardiology. I. Derivation of the integral equations Biophys. J. 7 443-62.

Baule G and McFee R 1965 Theory of magnetic detection of the heart's electrical activity J. Appl. Phys. 36 2060-73

van Dijk B W, de Munck J C and Spekreijse H 1990 The effects of conductivity parameters on estimates of the dipole sources of electric brain activity Electroenceph. Clin. Neurophysiol. submitted

Geddes L A and Baker L E 1967 The specific resistance of biological material-A compendium of data for the biomedical engineer and physiologist Med. Biol. Eng. 5 271-93

Geselowitz D B 1967 On bioelectric potentials in an inhomogeneous volume conductor Biophys. J. 7 1-11

Grynszpan F and Geselowitz D B 1973 Model study of the magnetocardiogram Biophys. J. 13 911-25

Jackson J D 1962 Classical Electrodynamics (New York: Wiley)

Morse P M and Feshbach H 1953 Methods of Theoretical Physics vols I and II (New York: McGraw-Hill) p 114

de Munck J C 1988 The potential distribution in a layer anisotropic spheroidal volume conductor J. Appl. Phys. 64 464-70

de Munck J C, van Dijk B W and Spekreijse H 1988 An analytic method to determine the effect of source modeling errors on the apparent location and direction of biological sources $J$. Appl. Phys. 63 944-56

Nicholson C and Freeman J A 1975 Theory of current source density analysis and determination of the conductivity tensor for an anuran cerebellum J. Neurophysiol. 38 356-67

Pethig R and Kell D B 1987 The passive electrical properties of biological systems: their significance in physiology, biophysics and biotechnology Phys. Med. Biol. 32 933-70

Plonsey R 1981 Magnetic field resulting from action currents on cylindrical fibers Med. Biol. Eng. Comput. $19311-5$

Plonsey R and Heppner D 1967 Considerations of quasistationarity in electro-physiological systems Bull Math. Biophys. $29657 \sim 64$

Rush S and Driscoll D A 1968 Current distribution in the brain from surface electrodes Anest. Analg. 47 717-23

Sarvas J 1987 Basic mathematical and electromagnetic concepts of the biomagnetic inverse problem Phys. Med. Biol. 32 11-22

Trahms L, Ernw S N, Trontelj Z, Curio G and Aust P 1989 Biomagnetic functional localization of a pheripheral nerve in man Biophys. J. 55 1145-53 